\title{
DETERMINAÇÃO DA RESISTÊNCIA EM FLEXÃO DE COMPÓSITOS DE MATRIZ POLIÉSTER REFORÇADOS COM FIBRAS DE BAGAÇO DE CANA-DE-AÇÚCAR*
}

Verônica Scarpini Candido ${ }^{1}$ Alisson Clay Rios da Silva² Sérgio Neves Monteiro ${ }^{3}$

\section{Resumo}

A utilização de fibras de naturais como agente de reforço tem sido amplamente estudada, pois é uma alternativa para o desenvolvimento de materiais sustentáveis. Assim esse trabalho tem por objetivo estudar o comportamento em flexão de compósitos de matriz poliéster reforçados com fibras de bagaço de cana de açúcar. Foram produzidos compósitos com adição de 0, 10, 20 e 30\% em volume de fibra e, depois, testados em flexão em 3 pontos. Os resultados mostraram que adição das fibras reduziu a resistência à flexão, porém, mesmo com uma queda dessa propriedade, os resultados são similares aos encontrados na literatura.

Palavras-chave: Compósitos, Fibra natural, Bagaço de cana-de-açúcar.

\section{DETERMINATION OF THE FLEXURAL STRENGTH OF REINFORCED POLYESTER MATRIX COMPOSITES WITH SUGARCANE BAGASSE FIBER}

\begin{abstract}
The use of natural fibers as a reinforcing agent has been widely studied as it is an alternative for the development of sustainable materials. Thus, this work aims to study the flexural behavior of polyester matrix composites reinforced with sugarcane bagasse fibers. Composites with $0,10,20$ and $30 \%$ by volume of fiber were produced and then tested in 3-point bending. The results showed that addition of the fibers reduced flexural strength, but even with a decrease of this property, the results are similar to those found in the literature.
\end{abstract}

Keywords: Composite, Natural Fiber,Sugarcane bagasse.

1 Bióloga, Doutora em Ciência dos Materiais, Professora Adjunta I, Faculdade de Engenharia de Materiais, Universidade Federal do Pará (UFPA), Ananindeua-PA, Brasil

2 Químico industrial, Doutor em Ciência dos Materiais, Professor Adjunto I, Faculdade de Engenharia de Materiais, Universidade Federal do Pará (UFPA), Ananindeua-PA, Brasil Brasil

3 Engenheiro de Materiais, PhD em Engenharia e Ciência dos Materiais, Professor, Programa de Pós-graduação em Engenharia de Materiais/Departamento de Engenharia mecânica e Materiais, Instituto Militar de Engenharia (IME), Rio de Janeiro, Rio de Janeiro, Brasil.. 


\section{INTRODUÇÃO}

Os materiais podem ser divididos, de maneira geral, em quatro grandes grupos: materiais metálicos, cerâmicos, poliméricos e compósitos [1]. Dentre esses, os compósitos é o grupo que vem apresentando um grande desenvolvimento científico [2].

Compósito é um tipo de material multifásico que exibe uma proporção significativa das propriedades de ambas as fases que o constituem, de tal modo que é obtida uma melhor combinação de propriedades [1]. Essas fases possuem composição e estrutura diferentes o que as tornam praticamente insolúveis entre elas [3].

Normalmente os compósitos são formados por uma fase contínua, também chamada de matriz e uma fase dispersa [1]. Tal definição pode ser considerada ampla e inclui ligas metálicas, co-polímeros de plástico, minerais e madeiras [4]. Um compósito polimérico reforçado com fibras consiste em uma fase matriz constituída de uma resina e a outra fase de fibra, que pode ser sintética ou natural.

O interesse atual em fabricar compósitos reforçados com fibras naturais se deve, entre outros fatores, a preocupação com a conservação ambiental e utilização de materiais renováveis [5]. Além disso, a produção desses materiais naturais, em substituição aos artificiais, pode representar uma diminuição no consumo de energia durante o seu processo de fabricação e, consequentemente, redução de custos de produção.

A utilização de fibras como reforço em matrizes poliméricas favorece aspectos ambientais e econômicos e resulta em novos materiais com propriedades mecânicas satisfatórias, o que permite diferentes aplicações [6]. Baixo custo, baixa densidade, resistência específica e módulo de elasticidade elevados [7], são algumas das características que favorecem a utilização em diversos setores industriais.

As fibras lignocelulósicas são constituídas basicamente de celulose, hemicelulose e lignina [5]. A celulose apresenta uma forma fibrosa que, juntamente com suas características estruturais, confere a fibra requisitos necessários para diversas aplicações na grande área de engenharia. Porem, a presença de propriedades não uniformes pode se uma desvantagem na utilização esse tipo de material [8].

A substituição das fibras sintéticas, como a de vidro e a de carbono, por fibras naturais tem sido observada no setor automobilístico na fabricação de tapetes, estofados e painéis [7]. Além da indústria automobilística, o setor da construção civil e de artigos esportivos também tem investido nesse novo tipo de material.

Um exemplo de fibra natural que pode ser utilizada para essa finalidade é a fibra de bagaço de cana-de-açúcar. A cana-de-açúcar (Saccharum officinarum), desde a época da colonização, é cultivada em larga escala pelo Brasil, mas foi com a explosão do álcool como combustível que esse vegetal se tornou conhecido e cultivado por todo o Brasil (ÚNICA, 2014). O centro-oeste, sul e sudeste juntos correspondem a $85 \%$ do cultivo da cana no país, deixando para o norte e nordeste os $15 \%$ restantes. De acordo com a FAO (Food and Agriculture Orgaization of the United Nations), cerca de $35 \%$ em peso da cana de açúcar produzida é constituída do bagaço, e na maioria das vezes inutilizado [9].

A utilização das fibras do bagaço da cana-de-açúcar para confecção de compósitos poliméricos apresenta vantagens comuns às outras fibras naturais e, além disso, contribui para a diminuição do desperdício dessa matéria-prima já que esse resíduo pode ser reutilizado. Dessa forma, nota-se que a utilização de 
materiais compósitos reforçados com fibras naturais é um ramo promissor na grande área de Ciência e Engenharia de Materiais e o estudo das propriedades desses materiais é de extrema importância. Assim, esse trabalho tem como objetivo estudar a resistência à flexão de compósitos de matriz epóxi reforçados com fibras de bagaço de cana-de-açúcar.

\section{MATERIAIS E MÉTODOS}

As fibras de bagaço de cana-de-açúcar foram lavadas em água corrente para retirada do excesso de açúcar e colocadas em estufa a $60^{\circ} \mathrm{C}$ durante $24 \mathrm{~h}$ para secagem. Posteriormente, foram desfiadas manualmente sendo o comprimento mínimo de $10 \mathrm{~cm}$.

Os corpos de prova para ensaio de flexão foram fabricados em matriz metálica utilizando-se resina poliéster e fibras de bagaço dispostas alinhadas longitudinalmente na matriz. O tempo de cura foi aproximadamente 24 horas a temperatura ambiente e depois, 2 horas em estufa a $100^{\circ} \mathrm{C}$. Após essa etapa, os corpos de prova foram lixados e cortados a fim de obterem as medidas solicitadas pelas normas para ensaios de flexão, ASTM D 790 [10].

O ensaio de flexão estática foi realizado em máquina de ensaios universais da Instron modelo 5582 utilizando a técnica de flexão em 3 pontos com uma taxa de deformação $0,5 \mathrm{~mm} / \mathrm{min}$ e uma capacidade de $100 \mathrm{kN}$ seguindo a norma ASTM D 790 no Laboratório de Materiais Avançados da Universidade Estadual do Norte Fluminense.

A região da fratura foi analisada utilizando-se um microscópio eletrônico de varredura da FEI modelo Quanta FEG - 250 no laboratório de microscopia do Instituto Militar de Engenharia.

\section{RESULTADOS E DISCUSSÃO}

Na figura 1 estão apresentadas as curvas típicas de Carga versus Extensão para resina poliéster e os compósitos, com adição de 10, 20 e 30\% em volume de fibras, obtidos a partir do ensaio de flexão estática.

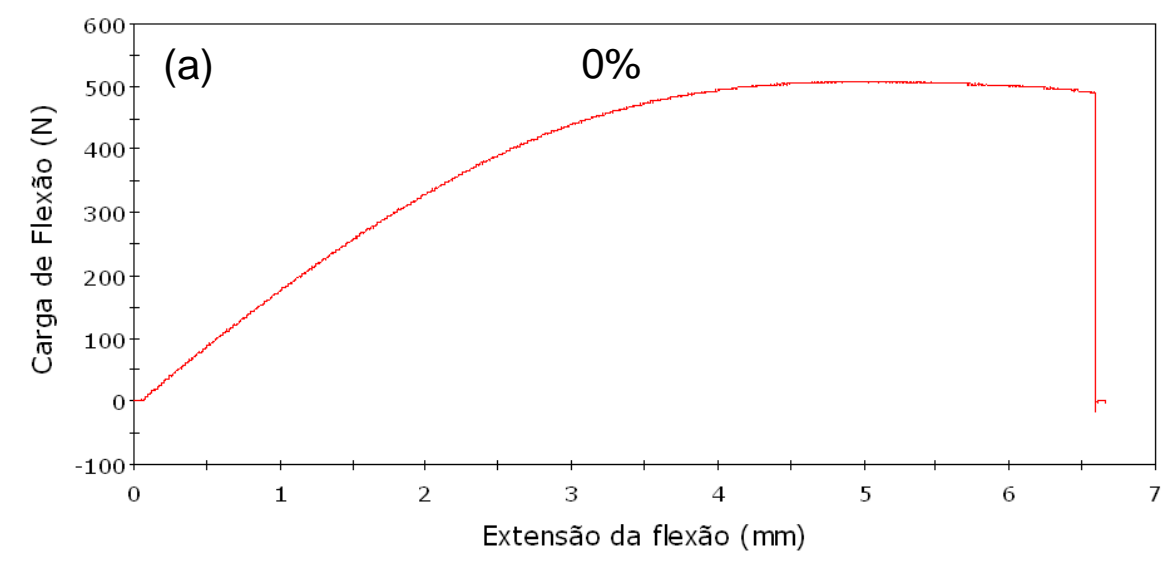



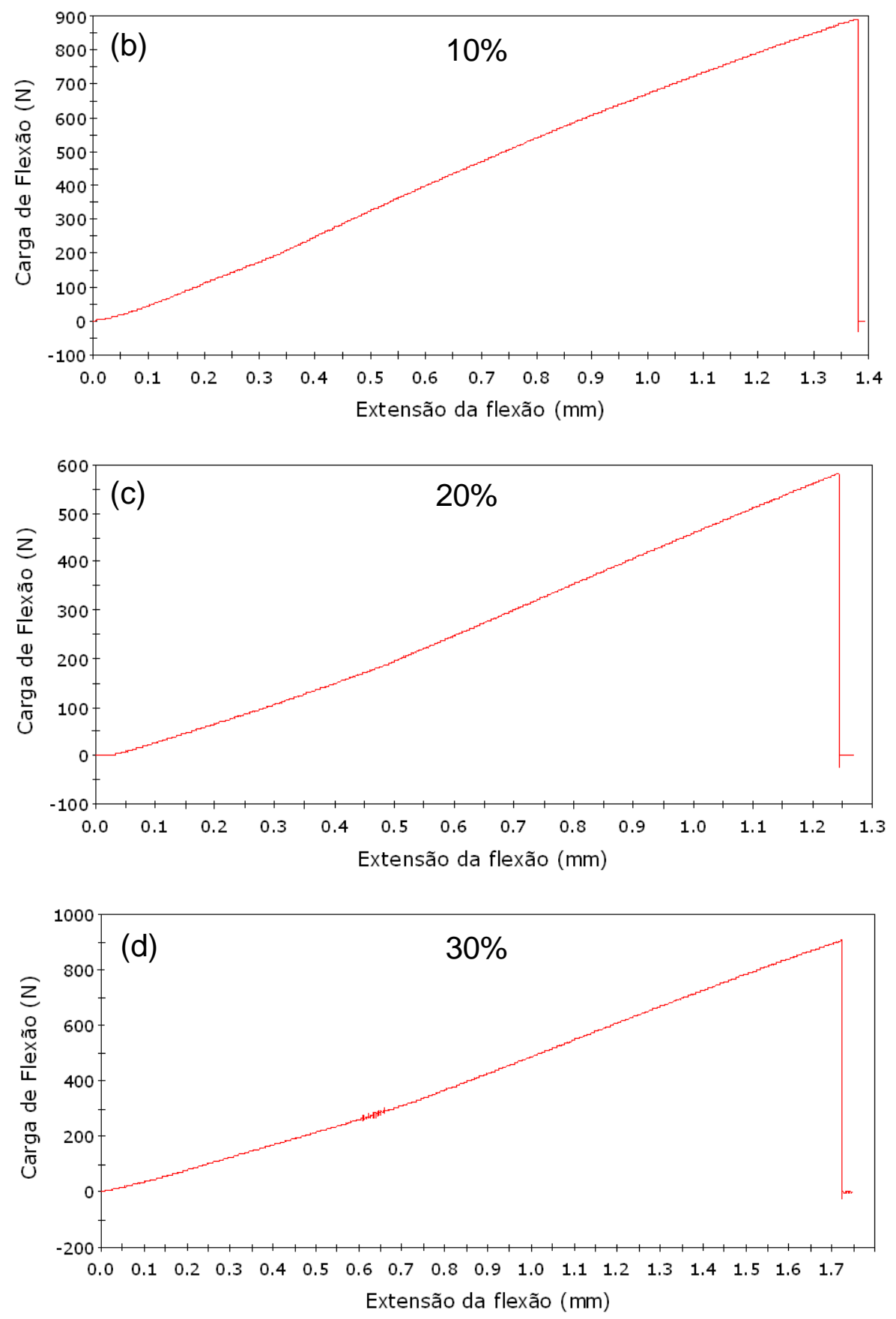

Figura 1. Curvas típicas de Carga versus Extensão do ensaio de flexão da resina poliéster (a) e dos compósitos com adição de 10 (b), 20 (c) e 30\% (d) em volume de fibra.

Pode ser observado na Fig. 1 (a) que a curva apresenta uma tendência parabólica com suave inclinação o que caracteriza certo comportamento plástico após o elástico. Diferentemente da resina poliéster pura, os compósitos apresentaram somente o comportamento elástico.

A partir dos resultados obtidos dos gráficos de Carga de flexão versus Extensão obteve-se os valores de resistência à flexão e do módulo de elasticidade, apresentados na tabela 1 . 
Tabela 1. Valores para resistência à flexão e módulo de elasticidade para a resina poliéster e os compósitos com adição de 10, 20 e 30\% em volume de fibra.

\begin{tabular}{ccc}
\hline $\begin{array}{c}\text { Fração em Volume das Fibras } \\
\text { de Bagaço de Cana-de- } \\
\text { açúcar } \\
(\%)\end{array}$ & $\begin{array}{c}\text { Tensão Máxima em Flexão } \\
\text { (MPa) }\end{array}$ & $\begin{array}{c}\text { Módulo Máximo de } \\
\text { Elasticidade em Flexão } \\
\text { (GPa) }\end{array}$ \\
\hline $\mathbf{0}$ & & $1,57 \pm 0,16$ \\
$\mathbf{1 0}$ & $96,65 \pm 5,77$ & $0,85 \pm 0,20$ \\
$\mathbf{2 0}$ & $42,30 \pm 3,60$ & $1,16 \pm 0,20$ \\
$\mathbf{3 0}$ & $42,78 \pm 7,00$ & $1,86 \pm 0,09$ \\
\hline
\end{tabular}

A Figura 2 apresenta a resistência à flexão e o módulo de elasticidade da resina poliéster e dos compósitos com adição de 10, 20 e 30\% em volume de fibra.

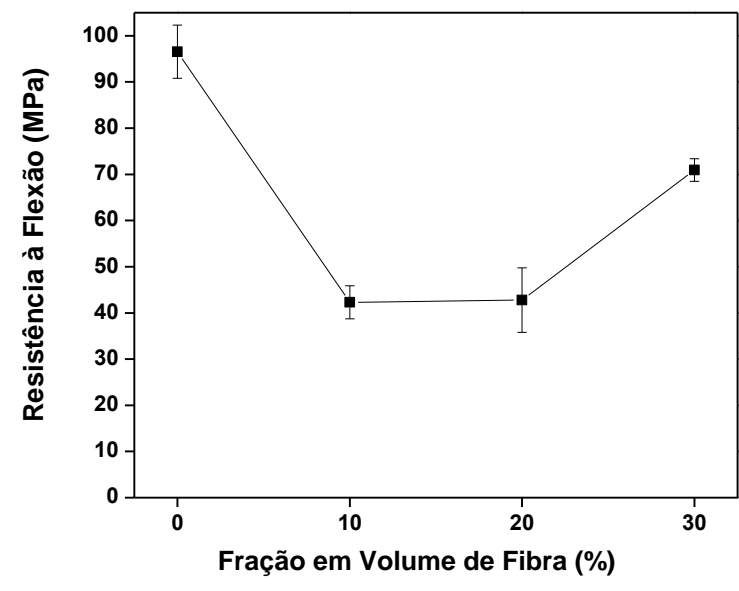

(a)

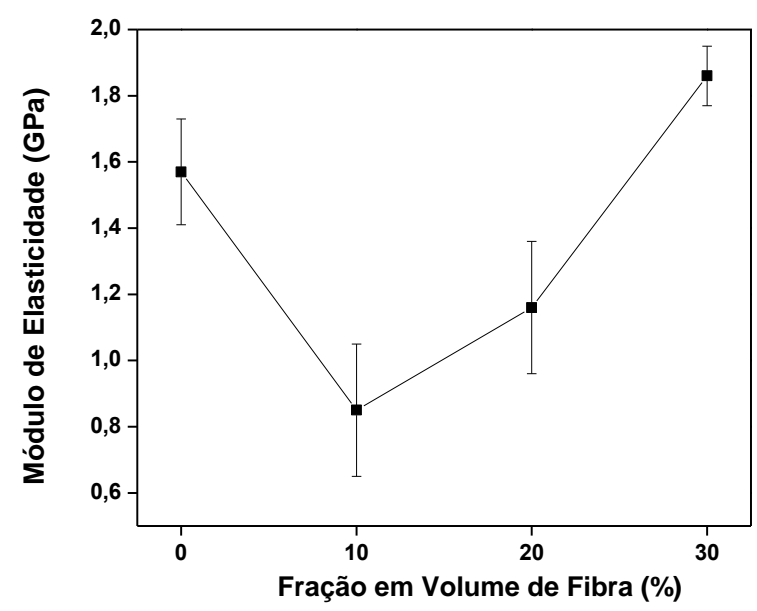

(b)

Figura 2. Resistência à flexão (a) e módulo de elasticidade (b) para o ensaio de flexão da resina poliéster e dos compósitos com adição de 10, 20 e 30\% em volume de fibra.

O ensaio de flexão revelou que adição da fibra de bagaço prejudicou tanto a resistência quanto o módulo de elasticidade. Esse fato pode estar associado à baixa adesão da fibra na matriz o que não favoreceu um reforço efetivo. Essa baixa adesão pode estar também associada ao elevado teor de lignina presente na fibra. Entretanto, considerando exclusivamente os compósitos, ocorre aumento tanto na resistência à flexão quanto no módulo de elasticidade com o aumento do volume incorporado de fibra de bagaço.

MONTEIRO et al., (1998) em um estudo sobre as propriedades de compósitos de poliéster incorporados com diferentes frações volumétricas de fibras de bagaço também observaram valores muito baixos para resistência à flexão dos compósitos. Os autores associaram tal resultado também ao alto teor de lignina, as imperfeições da fibra e à limpeza ineficiente que permitiu a permanência de açúcar superfície. Segundo MONTEIRO et al., (1998) [11], todos esses fatores dificultam a aderência da fibra na matriz. 
Em outro estudo sobre a influência do tratamento das fibras nas propriedades mecânicas de compósitos de poliéster reforçados com fibras de bagaço de cana-deaçúcar, VILAY et al. (2008) [12] verificaram que a resistência à flexão aumentou quando foram incorporadas fibras tratadas. Os autores atribuíram essa melhora a um aumento na adesão fibra/matriz.

Os resultados apresentados pelos compósitos de matriz poliéster indicam que a adição de fibras de bagaço de cana-de-açúcar pode comprometer o desempenho do material quando solicitado em flexão.

A figura 3 apresenta a micrografia da região da fratura da resina poliéster e dos compósitos com adição de 10, 20 e 30\% em volume de fibras com um aumento de 400x.
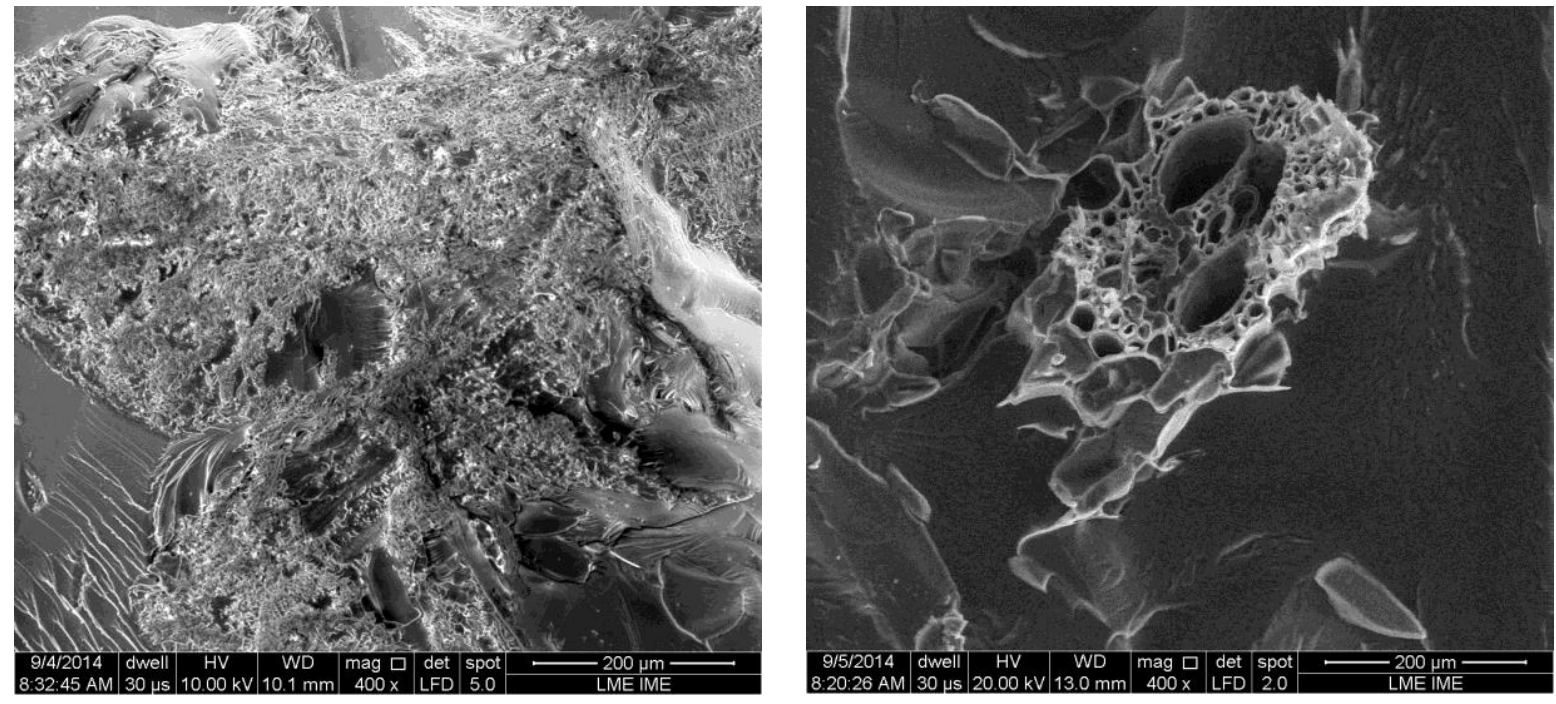


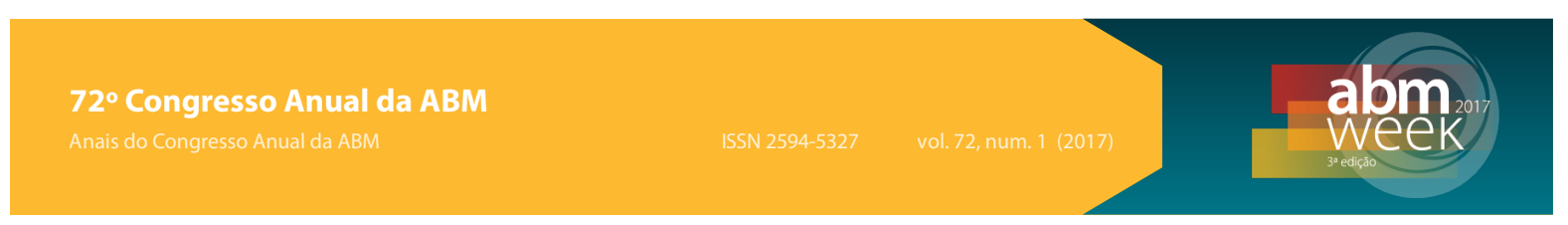

(a)

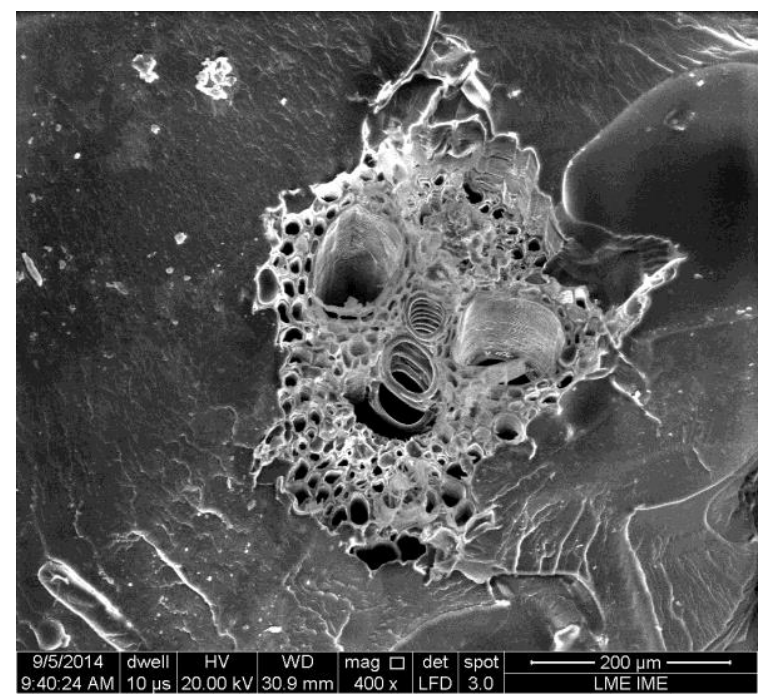

(c) (b)

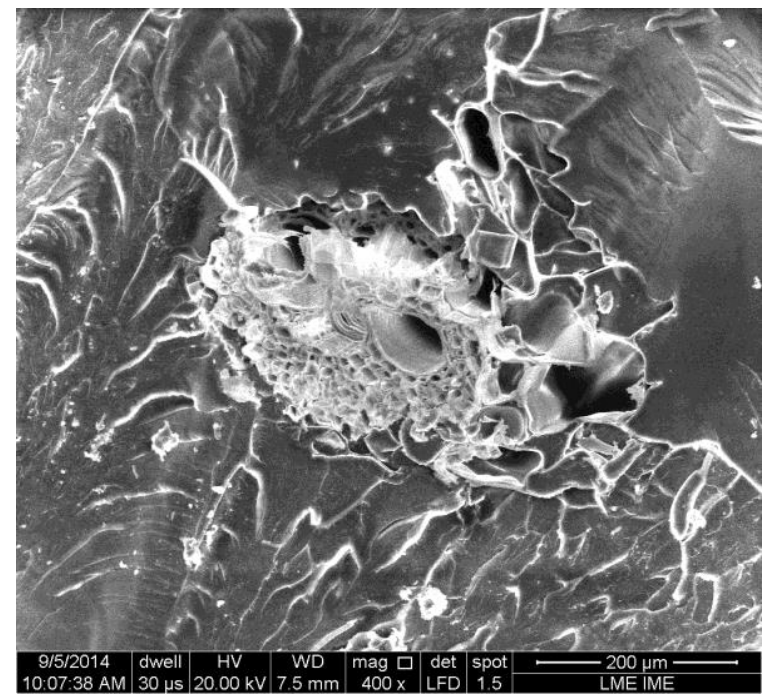

(d)

Figura 3. Micrografia da região da fratura por flexão estática obtida por MEV da resina poliéster (a) e dos compósitos com adição de 10 (b), 20 (c) e 30\% (d) em volume. 400x.

Nota-se que a resina poliéster e os compósitos possuem muitas áreas rugosas com poucas regiões lisas além de marcas de rio, o que é um indicativo de fratura frágil. Além disso, observa-se que as marcas de rio apresentam ponto de iniciação na interface fibra/matriz indicando que as fibras não aturam como um agente de reforço efetivo.

A observação da região da fratura permite inferir que interface gerada entre a matriz e a fibra de bagaço não é contínua, pois apresenta evidências de desacoplamento da fibra. MONTEIRO et al. (2005) [13] associaram esse fato à baixa tensão de cisalhamento encontrada para esse tipo de compósito.

De maneira geral, os valores de resistência à flexão apresentaram valores relativamente baixos quando comparados aos encontrados em compósitos reforçados com outras fibras naturais na literatura. MONTEIRO, et al. (2006) [14] ao investigarem o desempenho em flexão de compósitos reforçados com fibras de curauá encontraram valores próximos à $90 \mathrm{MPa}$ para as adições $30 \%$ em volume de fibra. DE DEUS et al. (2005) [15] ao incorporarem diferentes frações de fibras de piaçava em matriz poliéster alcançaram valores próximos à $70 \mathrm{MPa}$.

O baixo desempenho em flexão pode ser explicado pela baixa aderência, como citado anteriormente, e também, pelos defeitos inerentes à fibra natural. Além disto, em flexão, os corpos de prova estão submetidos a esforços mecânicos complexos. Isto pode, também, justificar a maior propensão a uma ruptura prematura

\section{CONCLUSÕES}

O estudo da resistência em flexão de compósitos de matriz poliéster reforçados com fibra de bagaço de cana-de-açúcar revelou que ao serem adicionadas fibras à matriz, os valores de resistência à flexão diminuíram, entretanto, os valores encontrados são muito similares aos encontrados na literatura. 
A diminuição da resistência pode estar associada à baixa adesão entre a fibra e a matriz o que acarreta em uma interface pouco resistente.

A resina poliéster e os compósitos apresentaram fratura frágil indicada na microscopia por marcas de rio e regiões em que as fibras foram arrancadas da matriz, confirmando a fraca adesão na interface fibra/matriz.

\section{REFERÊNCIAS}

1 WD Jr Callister. Ciência e Tecnologia de Materiais: uma introdução. 8 ed. Rio de Janeiro. Editora S.A. 705p, 2007.

2 FL Neto, LC Pardini. Compósitos Estruturais. Ed. São Paulo: Edgard Blucher, 313p., 2006.

3 KK Chawla. Composites Materials: Science and engineering. New York, Springer-Verlag, 1987.

4 SK Mazundar. Composites manufacturing: materials, product, and process engineering. CRC Press, New York, 2002.

5 KG Satyanarayana, JL Guimarães, F Wypych. Studies on Lignocellulosic fibers of Brazil. Part I: Source, production, morphology, properties and applications. Composites. Part A: applied science and manufacturing. 2007: 38, 1694-1709.

6 MJ John, S Thomas. Biofibres and Biocomposites. Carbohydrate Polymers. 2008: 71, 343-364.

7 SN Monteiro, FPD Lopes, AS Ferreira, DCO Nasciemnto. Natural fiber polymer matrix composites: cheaper, tougher and environmentally friendly. JOM. 2009: 61 (1) 17-22.

8 AL Leão, IH Tan, JC Craschi. Curaua fiber - A tropical natural fibers from Amazon - Potential and Application in Composites, In: International Conference on Advanced Composites, pp. 557-564, Hurghada, Egito, 1998.

9 UNICA - União das indústrias de cana-de-açucar. Disponível em: www.unica.com.br. Acessado em: janeiro de 2014

10 AMERICAN SOCIETY FOR TESTING MATERIALS, D 790 - Standard Test Method for Flexural Properties of Unreinforced and Reinforced Plastics and Electrical Insulating. ASTM, Pennsylvania: 2007.

11 SN Monteiro, RJS Rodriguez, MV de Souza, JRM D'Almeida. Sugarcane Bagasse Waste as Reinforcement in Low Cost Composites. Advanced Performance Materials 1998: 5, 183-191.

12 V Vilay, M Mariatti, R Mat Taib, M Todo. Effect of fiber surface treatment and fiber loading on the properties of bagasse fiber-reinforced unsaturated polyester composites. Composites Science and Technology. 2008:68, 631-638.

13 SN Monteiro, JRM D'Almeida, LAH Terrones, JRM Lopes. "Mechanical Strength of Polyester Matrix Composites Reinforced with Coconut Fiber Wastes", Rev. Mater.2005:10(4) 571-576.

14 SN Monteiro, RCMP Aquino, FPD Lopes, EA Carvalho, JRM D'Almeida. Comportamento mecânico e características estruturais de compósitos poliméricos reforçados com fibras contínuas e alinhadas de curauá. Revista Matéria. 2006: 11(3) 197-203.

15 JF de Deus, SN Monteiro, JRM D'Almeida. Effect of dryind molding pressure, and strain rate on the flexural mechanical behavior of piassava (Attalea funifera Mart) fiber-polyester composites. Polymer Testing. 2005: 24, 750-755.. 